\title{
Preliminary data on a computerized test of finger speed and endurance
}

\author{
BARRY A. TANNER and RICHARD L. BOWLES \\ Detroit Receiving Hospital and University Health Center, Detroit, Michigan \\ and Wayne State University, Detroit, Michigan
}

\begin{abstract}
We set out to develop a computer-assisted finger-tapping task (the T3) that would measure motor speed much like the Reitan test, but that would also measure endurance. Data were collected for a convenience sample on both the $\mathrm{T} 3$ and the Reitan finger-tapping test. Moderate and significant correlations were obtained between the T3 and the Reitan test for both hands. Mean scores for the first $50 \mathrm{sec}$ of the T3 were approximately 0.15 taps greater than the mean Reitan score for both the preferred and the nonpreferred hands, while the mean scores for the full 2 min of the T3 were 1.52 taps less than those of the Reitan test for the preferred hand, and 1.32 taps less for the nonpreferred hand. The mean for the last $40 \mathrm{sec}$ with the preferred hand averaged 3.93 taps (7.62\%) slower than for the first $40 \mathrm{sec}$, whereas for the nonpreferred hand, the difference was $5.12 \operatorname{taps}(11.15 \%)$. These results are consistent with our intent to develop measures of (1) relatively pure motor speed (the first 50 sec of the T3); (2) motor speed combined with endurance (the full 2 min of the T3); and (3) finger endurance (the first $40 \mathrm{sec}$ compared with the last $40 \mathrm{sec}$ of the T3).
\end{abstract}

The finger-oscillation test, part of Halstead's battery (Halstead, 1947), continues to be used in contemporary assessment (Reitan \& Wolfson, 1993), and is reported to be the most widely used neuropsychological measure (Lezak, 1983). The standard test involves having a subject tap a key which advances a mechanical counter, while the examiner times each 10 -sec trial with a stopwatch. The trials are repeated until five consecutive trials have been achieved with scores within a five-point range for each hand. The procedure readily lends itself to computerization (Whitfield \& Newcomb, 1992), which may result in improved data collection and reporting, as well as allow for entirely new measures of motor endurance. We have developed a computer-administered variant of the finger-tapping task, called $\mathrm{T} 3$, and report preliminary data comparing it with the Halstead-Reitan tapping test.

\section{METHOD}

\section{Subjects}

A nonclinical convenience sample of 40 subjects was recruited. Demographic characteristics of the sample are given in Table 1. The sample is more highly educated than the general adult population, with a mean of almost one year beyond college. The percentage self-identifying as left-handed is close to estimates for the general population.

\section{Materials}

Hardware. The tapper for the T3 is a PortaPoint miniature pointing device $(6.5 \times 4 \times 1 \mathrm{~cm})$. Unlike the Reitan tapper, which has a single lever mounted on the right, the PortaPoint contains two identical keys mounted on the left and right sides. The tapping de-

Correspondence should be addressed to B. A. Tanner, Psychology 9B, Detroit Receiving Hospital and University Health Center, 4201 St. Antoine, Detroit, MI 48201. vice is centered at the top of an EasyVue rigid mouse pad $(256 \times$ $308 \times 9.5 \mathrm{~mm}$ ), to which it is attached by Velcro fasteners. The keys on the PortaPoint are larger than those on the Reitan, and move much less (about $2 \mathrm{~mm}$ vs. approximately $15 \mathrm{~mm}$ ).

Images of two hands in the desired position are fixed under the clear tracking surface of the EasyVue, clarifying for the subject how each hand should be held while tapping. The hands are labeled LEFT and RIGHT in large letters, and the clear surface is fixed in place with vinyl tape. The PortaPoint communicates with an IBMcompatible PC through the PS/ 2 mouse port. Instructions for the task are provided through headphones or speakers attached to a sound board.

Software. The verbal instructions are contained in WAV files, accessed through calls to the Windows API. The program, which is written in Microsoft Visual BASIC 3, instructs the subject in the placement of both hands, referring to the images of the hands on the EasyVue. Through calls to the Windows API, responses to the appropriate key are isolated, with the right key being used for the right hand and the left key for the left hand. The program will not continue until the subject presses the indicated key. Following a 10sec practice trial, the subject's attention is verbally directed to the monitor, where each of 12 text boxes is pointed to in turn. These boxes are used to provide ongoing feedback concerning the subject's performance and to allow comparison of performance, both from one $10-\mathrm{sec}$ interval to another and from one hand to the other. The subject is then instructed to tap as fast as possible without lifting his or her finger off the key or hand off the mouse pad. Consistent with the Reitan procedure, the $\mathrm{T} 3$ does not begin timing until the first keypress, as this is not intended to be a measure of response latency. The subject is instructed to stop tapping with the right hand when $2 \mathrm{~min}$ have elapsed, and the procedure is repeated with the left hand. Whereas the Reitan procedure allows for brief rest periods between $10-\mathrm{sec}$ trials, with a mandatory rest after the third trial, the 12 trials of the T3 are continuous, although the data are recorded for discrete 10 -sec blocks.

\section{Procedure}

Each subject completed a slightly modified version of the Reitan tapping test, and the standard T3. The Reitan procedure was modified in that only five trials were given, even if the criteria of 
Table 1

Sample Demographics

\begin{tabular}{lcccccccc}
$\begin{array}{c}\text { Sample } \\
\text { Size }\end{array}$ & \multicolumn{3}{c}{ Age (years) } & & Female & Left-Handed & \multicolumn{2}{c}{ Education (years) } \\
\cline { 2 - 4 } & $M$ & $S D$ & Range & $(\%)$ & $(\%)$ & & $M$ & $S D$ \\
\hline 40 & 32.80 & 15.69 & $12-70$ & 50 & 15 & 16.93 & 2.81 \\
\hline Note- $M$, mean; $S D$, standard deviation. & & & & &
\end{tabular}

five consecutive trials within five taps was not met. The subjects alternated in terms of whether they took the Reitan test or the T3 first. They were told that we were collecting pilot data to compare a new computerized measure with the existing manual measure. The T3 records data to a disk file, to which the Reitan data were added by the examiner after first recording them on paper. Since we were concerned with how similarly the two approaches measure finger speed, we examined this with correlation coefficients and by comparing means.

\section{RESULTS}

Examination of the mean taps per $10 \mathrm{sec}$ for the two lengths of the T3 and for the Reitan test suggests that the short version of the $\mathrm{T} 3$ provides results more like that of the manual test. Table 2 shows that while the mean for the short $\mathrm{T} 3$ is no more than 0.15 taps greater than that for the Reitan test for both the preferred and nonpreferred hand, the mean for the full T3 is more than a full tap slower for each hand. Jarvis and Barth (1984) state that the preferred hand is normally about $10 \%$ faster than the nonpreferred hand, while all of our measures show a speed advantage for the preferred hand that averages about $11 \%$. The correlation coefficients for the short T3, the full T3, and the Reitan are displayed in Table 3. All correlations are significant beyond the .01 level (Cohen \& Cohen, 1975). While we had expected the Reitan and

Table 2

Mean Taps Per 10 Sec and Preferred-Hand Advantage

\begin{tabular}{lccc}
\hline & \multicolumn{3}{c}{ Test } \\
\cline { 2 - 4 } & & \multicolumn{2}{c}{ T3 } \\
\cline { 2 - 4 } & Reitan & Short & Full \\
\hline Preferred & 50.66 & 50.77 & 49.30 \\
Nonpreferred & 45.13 & 45.27 & 43.61 \\
Advantage for preferred hand (\%) & 11.24 & 10.83 & 11.54 \\
\hline
\end{tabular}

Table 3

Correlation of the Short and Full T3 With the Reitan

\begin{tabular}{llc}
\hline & \multicolumn{2}{c}{ T3 } \\
\cline { 2 - 3 } & Short & Full \\
\hline Preferred & .607 & .612 \\
Nonpreferred & .648 & .693 \\
\hline
\end{tabular}

Note-All correlation coefficients are significant at $p<.01$.

Table 4

Mean Taps for First and Last 40 Sec of T3

\begin{tabular}{lcc}
\hline & First $40 \mathrm{Sec}$ & Last $40 \mathrm{Sec}$ \\
\hline Preferred & 51.54 & 47.61 \\
Nonpreferred & 45.94 & 40.82 \\
\hline
\end{tabular}

Note--Means in the same row differ at $p<, 01$.
$\mathrm{T} 3$ to be significantly related, these results take us beyond that assumption.

We included a full 2 min of tapping in the T3 in an attempt to add an endurance factor to the test. Table 4 compares the mean taps for the first and last $40 \mathrm{sec}$ with each hand. As expected, the last $40 \mathrm{sec}$ showed a slowing, which we believe is due to fatigue; $t$ tests for related measures revealed that this hypothesized endurance or fatigue factor took a toll on both the nonpreferred hand $[t(39)=$ $9.74, p<.01]$ and the preferred hand $[t(39)=7.55, p<$ $.01]$. The effect of fatigue tended to be less for the preferred hand, which may have resulted from most subjects using their preferred hand more often and therefore having greater strength and endurance in that hand.

\section{DISCUSSION}

The results indicate some success in developing a computer-based measure of finger speed and endurance. The first $50 \mathrm{sec}$ of the $\mathrm{T} 3$ appear to provide a relatively pure measure of motor speed, while the difference between the first and the last $40 \mathrm{sec}$ seems largely to measure endurance. It is unlikely that the existing norms for the Reitan procedure can be used with the T3, given that they are not identical measures of motor speed. Norms will also be needed for the T3 endurance score.

The visual feedback provided on the monitor was intended to encourage maximal performance, and also to facilitate future studies of suboptimal performance in which the feedback is manipulated. Although the results are encouraging, the ultimate clinical utility of this measure will rest in its ability to discriminate between various populations (e.g., those with anterior vs. those with posterior lesions), and such studies are being planned.

\section{REFERENCES}

CoHEN, J., \& CoHEN, P. (1975). Applied multiple regression/correlation analysis for the behavioral sciences. Hillsdale, NJ: Erlbaum. HalsteAD, W. C. (1947). Brain and intelligence. Chicago: University of Chicago Press.

JARVIS, P. E., \& BARTH, J. T. (1984). Halstead-Reitan test battery: An interpretive guide. Odessa, FL: Psychological Assessment Resources.

LEZAK, M. D. (1983). Neuropsychological assessment (2nd ed.). New York: Oxford University Press.

ReItAN, R. M., \& Wolfson, D. (1993). The Halstead-Reitan neuropsychological test battery: Theory and clinical interpretation (2nd ed.). S. Tucson, AZ: Neuropsychology Press.

WhITFIELD, K., \& NewCOMB, R. (1992). A normative sample using the Loong computerized tapping program. Perceptual \& Motor Skills, 74, 861-862.

(Manuscript received November 23, 1994; revision accepted for publication January 20, 1995.) 